\title{
Política ambiental chilena y política indígena en la coyuntura de los tratados internacionales (1990-2010) ${ }^{1}$
}

\author{
Sara Zelada Muñoz
}

Universidad de Los Lagos, Puerto Montt, Chile. Email: sarazel@gmail.com.

\section{James Park Key}

Universidad de Los Lagos, Osorno, Chile. Email:jpark@ulagos.cl, parkchile@gmail.com

Resumen: Se analizan las políticas medio ambientales e indígenas durante el período 1990- 2010 de gobiernos de la Concertación, y los tratados internacionales sobre el medio ambiente que inciden en el uso de recursos naturales en territorios huilliche. Se concluye que la política pública medioambiental, por su naturaleza reactiva, en el contexto de los mercados globales, se ha visto sobrepasada por la hegemonía del poder de las transnacionales que invierten en los commodities forestal, minero, agropecuario, amparadas por una legislación ambiental débil y un discurso político obsecuente.

Palabras clave: legislación ambiental, política pública, derechos indígenas, recursos naturales, biodiversidad.

\section{Chilean environmental policy and indigenous policy at the juncture of international treaties (1990-2010)}

Abstract: Indigenous and environmental policies are analyzed during the period 1990-2010, during the governments of the 'Concertación', and those international treaties relating to the environment which may have direct incidence on the natural resources in huilliche territory. The conclusion is that public environmental policy, due to its reactive nature, in the context of global markets, has been overcome by the hegemonical power of transnational corporations who invest in forestry, mining and agro commodities, all of which are sheltered by weak environmental legislation and an obsequious political discourse.

Key words: environmental legislation, public policy, indigenous rights, natural resources, biodiversity.

\section{Política ambiental do Chile e política indígena na conjuntura dos tratados internacionais (1990-2010)}

Resumo: Analisamos as políticas ambientais e indígenas durante o período de 1990 - 2010 de governos da Concertación, os tratados internacionais sobre o meio ambiente que afetam o uso de recursos naturais em territórios huilliche. Conclui-se que a política pública ambiental, por sua natureza reativa, no contexto dos mercados globais foi ultrapassada pela hegemonia do poder transnacional de investir em commodities silvicultura, mineração, agricultura, protegida pela 
legislação ambiental fraca eo discurso político obsequioso.

Palavras-chave: direito ambiental, políticas públicas, direitos indígenas, recursos naturais, biodiversidade.

\section{Introducción}

Chile posee una gran riqueza de ambientes marinos, costeros, terrestres e insulares, en donde han evolucionado un número de especies vegetales y animales únicas en el mundo. El aislamiento geográfico de nuestro país, debido a sus barreras geográficas y climáticas, ha determinado que éste sea considerado como una isla biogeográfica, con una biodiversidad de un alto endemismo en ecosistemas muy variados.

En el sur de Chile se presentan bosques de clima templado-húmedo que se destacan en relación a los bosques templados del hemisferio norte, como consecuencia de la heterogeneidad de ambientes en que se desarrollan, producto de las variaciones altitudinales y latitudinales que -incrementadas por el vulcanismo, las glaciaciones y los deslizamientos de tierra- contribuyeron a elevar la riqueza biológica de estos bosques (Catalán y Ramos, 1998).

Los bosques nativos proveen servicios ambientales tales como: la regulación y provisión de agua, la captura de carbono, la conservación de suelos y de la diversidad biológica (Lara, Urrutia, Little y Martínez, 2010, 3); poseen, además, valor económico, social, cultural y religioso. La selva valdiviana en la X Región de Chile -así como todo el territorio americanoperteneció a los pueblos originarios que desde tiempos inmemoriales desarrollaron su cultura y economía, de maneras diversas y sustentables (Toledo, 2003:113; Bengoa, 1996: 17), reconocido por la Cumbre de Río de Janeiro². Estos recursos se encuentran en la actualidad seriamente amenazados por la profundización de la economía de libre mercado con proyectos de inversión que se realizan principalmente en territorios indígenas en donde se han generado agudos conflictos.

En la década de los setenta se configuran en Chile las primeras políticas medioambientales en respuesta a las iniciativas de protección del ambiente que comienzan a formalizarse en occidente para enfrentar el deterioro -ya entonces evidente- que experimenta la naturaleza como consecuencia del modelo neoliberal de desarrollo económico. La National Environmental Policy Act en 1969 introduce el sistema de evaluación ambiental, iniciando una etapa en que instituciones y principios jurídicos apuntan a uniformar en alguna medida el Derecho Ambiental que se aplica en occidente (Olivares, 2010: 3).

Siguiendo esta tendencia, en la Universidad de Chile, se conforman los primeros grupos de investigación y docencia en el campo de Ecología y 
Medio Ambiente. En 1967 se crea la Comisión Nacional de Investigación Científica y Tecnológica y se generan los primeros fondos para el desarrollo de proyectos de investigación que se formalizará oficialmente en instituciones como la denominada FONDECYT. En 1968 nace el Comité de Defensa de Flora y Fauna (CODEFF) con el propósito de luchar contra los problemas derivados de la explotación de los recursos naturales y, a la fecha, es una de las organizaciones más importantes del país. Sin embargo -con una salvedad ${ }^{3}$ - no se dio en este período una preocupación por el medio ambiente, los recursos naturales, la contaminación y los perjuicios consustanciales al desarrollo de la actividad minera (Camus y Hajek, 1998).

En 1967 se promulga la Ley de Reforma Agraria. En 1972 el gobierno de Salvador Allende promulga la Ley $\mathrm{N}^{\circ} 17.729$ que establece la posibilidad de restituir tierras a los indígenas utilizando para ello el mecanismo contemplado en la reforma agraria, y crea el Instituto de Desarrollo Indígena a fin de promover su desarrollo económico, social, cultural e integral.

Entre 1973 y 1989 -durante el gobierno militar- se impone en el país la política económica fundada en el libre mercado, caracterizada por una desregulación de la economía y apertura al comercio exterior, así como la progresiva privatización de las empresas estatales y la inversión extranjera en la explotación de los recursos naturales (Altieri y Rojas, 1999: 56). El Decreto Ley No 701 de 1979 sobre fomento forestal, estableció la bonificación del fisco a las plantaciones particulares y la privatización de empresas forestales estatales, el que logró sólo en cinco años (1985-1989) que el Estado de Chile vendiera más de treinta grandes empresas, con enormes pérdidas monetarias debido a los bajos precios de venta negociados (Monckeberg, 2001 citado en Frêne y Núñez, 2010: 26). El Código de Aguas de Chile (1981) y el Código de Minería (1983) permitieron entregar títulos individuales sobre estos recursos, de modo que fuesen apropiados por las compañías forestales y mineras. El Código de Aguas estableció que el Estado otorgaría derechos para usar el agua, pudiendo ser transados en el mercado y transferidos a personas ajenas a una comunidad indígena; para este efecto las aguas debían inscribirse a título individual (Castro, 2003: 524).

Un balance de las actividades productivas primarias del período 7389, permite apreciar que los conflictos sociales y ambientales desencadenados por las forestales y mineras -en el marco de la tendencia internacional hacia la protección del medio ambiente y el desarrollo sustentable- propiciaron iniciativas gubernamentales como la ratificación de la Convención sobre Comercio Internacional de Especies Amenazadas de Fauna y Flora Silvestres (CITES) en 1975, la Ley sobre Industria Pesquera y sus derivados (1983), la creación de la Comisión Nacional de Ecología (1984), la Comisión de Legislación del Medio Ambiente en 1985 (que no dio resultados), la creación del Sistema Nacional de Áreas Silvestres (1984) y la Regulación para la pesquería pelágica en I, II y VIII Regiones (1986), entre otras. La Convención Ramsar fue aprobada en Chile como Ley de la República en septiembre de 1980 y promulgada como tal a través del Decreto Supremo N $^{\circ} 771 / 1981$, del Ministerio de Relaciones Exteriores. 
Sin embargo, estas medidas medioambientales y las del período siguiente han resultado inconsistentes con las disposiciones privatizadoras de la Constitución de 1980 y otros cuerpos jurídicos, tales como el Código de Aguas, el Código Minero y la Ley de Pesca, antes mencionados, que amparan la apropiación por no indígenas de recursos mineros, agua, recursos pesqueros y otros recursos naturales que se encuentran en tierras indígenas (Aylwin, 2002: 20).

En la región de Los Lagos (X Región), la expansión de las compañías forestales y de celulosa, las hidroeléctricas, la salmonicultura (entre otras), han ocasionado graves problemas ambientales, algunos de los cuales son: contaminación de ríos y lagos por aguas servidas y residuos industriales, especialmente en lago Llanquihue; erosión de suelo por mal manejo de actividades agrícolas y forestales; empobrecimiento de praderas naturales por sobrepastoreo en la isla de Chiloé; irracional explotación y destrucción de bosques nativos; peligro de extinción de especies marinas; escasa reforestación y reforestación con monocultivos, especialmente de pino insigne; uso descontrolado de plaguicidas en la agricultura; excesivo uso de la vegetación para consumo de leña que especialmente afecta a especies nativas, entre otros (Hajek., Gross. y Espinoza, 1990: 224).

En Chiloé, la industria salmonera ha tenido un desarrollo intensivo a partir de los años ochenta, afectando de modo radical a las comunidades mapuche-huilliche que han visto grandes porciones de su territorio severamente afectadas por la contaminación, la desaparición de la biodiversidad marina, vital como recurso alimenticio, y las restricciones de acceso a los bancos naturales de recursos pesqueros por la concesión de éstos a privados (Morales, Tamayo y Cox, 2010: 5)

\section{Legislación ambiental y política indígena en el periodo 1990-2010}

Chile ha sido país pionero en Latinoamérica en cuanto al enorme impulso dado a los sectores exportadores de recursos naturales, con la drástica reforma económica de los años setenta. Esto significó que muy pronto el sector exportador se viese apremiado por los estándares ambientales exigidos por los mercados globalizados. Rojas, Sabatini y Sepúlveda (2003: 22) manifiestan que estas acciones ambientales no fueron acompañadas de avances en legislación, institucionalidad y políticas públicas, sino que solo fueron tomadas por el Estado en su afán por apoyar las exportaciones. Así el tema ambiental se ve incorporado de manera perentoria a la agenda política de los gobiernos democráticos a partir de los noventa, lo mismo sucede con los derechos indígenas amparados por los tratados internacionales. La relación entre lo ambiental, lo étnico y lo político no ha sido casual, se trata de un problema sistémico que no puede ser abordado con éxito, sin una visión integral. 


\section{Período de la Presidencia de Patricio Aylwin (1990-1994)}

Las elecciones presidenciales de 1989 y la instalación del gobierno demócrata-cristiano de Patricio Aylwin permiten acotar un nuevo período en la historia ambiental de Chile. Se acusaba al gobierno del general Pinochet de haber contraído una deuda ambiental debido a su despreocupación prácticamente total por la temática, y que Chile había sufrido en los últimos años un marcado deterioro de sus recursos naturales y una creciente presencia de fenómenos puntuales de contaminación. La dispersión de leyes e instituciones con relevancia ambiental hacía imposible una política coherente y coordinada respecto del medio ambiente (Asenjo, 1990 en Camus y Hajek, 1998).

En 1991 el Congreso de Pueblos Indígenas reafirmó los puntos principales del proyecto de Ley Indígena, y éste se envió al Congreso simultáneamente con otros tres proyectos: el proyecto de Reforma Constitucional, que tenía por objeto lograr mayor grado de reconocimiento jurídico de los pueblos indígenas, de manera que Chile se reconociera como país pluriétnico y pluricultural; el proyecto de ratificación del Convenio 169 de la OIT sobre pueblos indígenas y tribales; y el proyecto que crea el Fondo de Desarrollo de los Pueblos Indígenas de América Latina y El Caribe. Estas cuatro iniciativas debían ser aprobadas conjuntamente, por hallarse imbricadas en el contexto nacional e internacional a favor del respeto a los derechos de los pueblos originarios, pero solamente fue aprobado -aunque con modificaciones y cambios sustanciales- el proyecto de Ley Indígena, y se ratificó el Convenio Constitutivo del Fondo de Desarrollo Indígena. En 1993 se aprueba la Ley Indígena ( $\left.\mathrm{N}^{\circ} 19.253\right)$ que establece Normas sobre Protección, Fomento y Desarrollo de los Indígenas, y crea la Corporación Nacional de Desarrollo Indígena /(CONADI), sin embargo, los proyectos de reforma constitucional que hubiesen hecho posible garantizar los derechos indígenas, no prosperaron (Carrasco, 2008: 319).

La ley Indígena dispone que es deber de la sociedad en general y del Estado en particular, a través de sus instituciones, respetar, proteger y promover el desarrollo de los indígenas, sus culturas, familias y comunidades, adoptando las medidas adecuadas para tales fines y proteger las tierras indígenas, velar por su adecuada explotación, por su equilibrio ecológico y propender a su ampliación (Art. $1^{\circ}$ ). Aylwin (2002:12-17) destaca los elementos positivos de la ley: el reconocimiento de las tierras ancestrales, su protección jurídica y establecimiento de un fondo especial -el fondo de tierras y aguas- para proveer su ampliación; el establecimiento de Áreas de Desarrollo Indígena (ADI) y la promoción de programas de educación ambiental bilingüe en las áreas indígenas; el derecho a ser escuchados y considerados en su opinión al tratarse materias que les atañen, etc. Sin embargo, la nueva ley negó a los indígenas su carácter de pueblos originarios señalándolos como etnias, eliminó el impedimento del proyecto de ley para trasladar a los indígenas de sus tierras y de consultar dicho traslado, modificó el deberá contemplar la participación de las comunidades existentes en las Áreas de Desarrollo Indígena (ADI) en la administración de 
las áreas silvestres protegidas ubicadas reemplazándolo por el ambiguo se considerará su participación, eliminó la figura de los jueces de paz indígenas encargados de impartir justicia de menor cuantía al interior de la comunidad, también eliminó del proyecto de ley la norma que establecía derechos preferentes para los titulares de tierras indígenas, derechos de agua, mineros, de aprovechamiento y manejo de recursos forestales, uso de riberas, en tierras indígenas y aguas colindantes, a la par de otros cambios que le restaron a la ley su sentido original, haciéndola inaplicable

Foerster y Vergara (2000:13) constatan que, a pesar de los logros alcanzados con la promulgación de la ley indígena y la mayor sensibilidad hacia la sociedad mapuche y sus demandas, no ha habido una transformación sustantiva de los modos de relacionarse con ella de parte del Estado, ni de las empresas, ni menos aún de la siempre influyente elite dominante y su vocero, El Mercurio. Los mapuche buscan un reconocimiento etnonacional, y en el Gobierno, el reconocimiento es étnico y ciudadano, aunque con tendencia a retomar una política campesinista, en tanto que para la elite el único reconocimiento posible es el que se ofrece a todos los demás chilenos, insistiéndose además en la necesidad de restaurar el estado de derecho, y llevar a cabo la deseada pero nunca concluida integración de los mapuche a la nación chilena Por su parte Aylwin (2000: 99) estima que todos los esfuerzos que CONADI y otros organismos realicen para hacer efectiva la protección de la Ley Indígena a las tierras calificadas como tales, resultarán en vano si no se concretan las modificaciones legales que permitan vincular dichas tierras con recursos tales como el agua, los recursos mineros y pesqueros que se encuentran en ellas, en concordancia con la tendencia internacional y comparada existente en esta materia.

\section{Período de la Presidencia de Eduardo Frei (1994-2000)}

El 9 de marzo de 1994 se publica en el Diario Oficial la Ley $\mathrm{N}^{\mathrm{o}}$ 19.300 que otorga una nueva estructura a la Comisión Nacional del Medio Ambiente (CONAMA), entidad que incorpora la dimensión ambiental en la gestión de los ministerios. Camus y Hajek (1998) hacen notar que la perspectiva ecologista rechazó el proyecto de Ley de Bases del Medio Ambiente denunciando que el proyecto de ley constituía un retroceso, y afirmando que en la cúpula de la izquierda gobiernista no existía ninguna conciencia para proteger la naturaleza.

Los fundamentos de la política ambiental de gobierno fueron expuestos en el documento Una Política Ambiental Para El Desarrollo Sustentable, aprobado por el Consejo Directivo de Ministros de CONAMA en la Sesión del 9 de enero de 1998. El documento sostiene que la política ambiental se fundamenta en: 1) la calidad de vida de las personas; 2) la complementariedad entre el desarrollo socioeconómico y la sustentabilidad ambiental; 3) la equidad social y superación de la pobreza. Enseguida, se enumeran sus diez principios conductores, entre los que destacamos, las políticas públicas ambientalmente sustentables, involucrando transversalmente a todos los sectores del gobierno; la participación de la ciudadanía 
organizada, hacia una gestión ambiental legítima, transparente y socialmente consensuada; la sustentabilidad, que incorpora el concepto de equidad intergeneracional; y la responsabilidad del causante, la prevención, la estabilidad, el gradualismo y el cumplimiento de los acuerdos internacionales.

Paradojalmente, surgen relevantes conflictos ambientales que desencadenan una serie de manifestaciones, declaraciones y protestas de las organizaciones indígenas, de los ecologistas y estudiantes, quienes acusan al gobierno de falta de voluntad política para equilibrar el desarrollo económico con la protección del medio ambiente y la democratización. Tales fueron el proyecto GasAndes, impulsado por las empresas chilenas Gasco y Chilgener, cuyo objetivo consistía en transportar gas natural desde la Provincia de Neuquén en Argentina para abastecer a la Región Metropolitana; la Forestal Trillium y el proyecto Río Cóndor en Magallanes; la construcción de la Central Hidroeléctrica Pangue y la Central Ralco, en el cauce del río Bío Bío.

En marzo de 1997 fue inaugurada la represa Pangue, y el 24 de septiembre de 1997 el Consejo Directivo de la CONAMA emitió su Resolución final favorable a la Empresa Nacional de Electricidad S.A. (ENDESA) ${ }^{4}$ para el proyecto Ralco, a pesar de los informes adversos de diversas instituciones académicas, organizaciones indígenas, ecologistas y de la CONADI, que se oponían a la construcción de Ralco por las numerosas y profundas deficiencias del estudio de impacto ambiental, y los impactos invaluables a la cultura pehuenche ancestral. Luego de que los tres principales directivos de la Corporación Nacional Indígena fueran destituidos de sus cargos por el presidente Frei, se dio vía libre al proyecto, los pehuenches fueron desalojados de sus tierras, relocalizados en tierras altas de veranada que se cubren de nieves en el invierno, e inundados sus cementerios y sitios arqueológicos, quebrantando la ley indígena y los tratados internacionales.

\section{Período de la Presidencia de Ricardo Lagos (2000-2006)}

El programa de gobierno de Ricardo Lagos - que se identificaba con el slogan el progreso con igualdad- se comprometía con la protección del medio ambiente y con un amplio reconocimiento a los pueblos indígenas, sus tradiciones, lenguajes y formas de expresión cultural que habría de concretarse en la política de nuevo trato a favor de la no discriminación racial a través de una nueva institucionalidad política y jurídica en favor de la participación de los indígenas en la sociedad chilena y respecto del cumplimiento de sus derechos. Es así como a comienzos del 2001 se crea la Comisión Verdad Histórica y Nuevo Trato, en medio de la conflictividad existente en territorio mapuche por recuperación de tierras. Aylwin (2002: 35) sostuvo entonces, que no se podía esperar a que la Comisión concluyera su trabajo para resolver la situación en el sur del país, con su costo social, en particular para los mapuche, enorme.

El Ministerio de Obras Públicas (MOP) decidió, a mediados de 2001, 
continuar con el proyecto Carretera de la Costa que fuera resistido a nivel local e internacional. El tramo sur de esta carretera atraviesa territorios que han ocupado ancestralmente las comunidades huilliches. El proyecto implica omitir la aplicación de disposiciones de varias normas vigentes, como las contenidas en la Ley de Bases del Medio Ambiente, la Ley Indígena e incluso en la misma Constitución de la República, que ordenan la participación de las comunidades y sus organizaciones tradicionales en relación a la ejecución de proyectos en sus territorios (Correa, Catalán y Paillamanque 2001: 1).

\section{Período de la Presidencia de Michelle Bachelet (2006-2010)}

El Programa de Gobierno de Michelle Bachelet asumió diez compromisos con los grupos ecologistas respecto a la política medioambiental de su gobierno, en el llamado Acuerdo de Chagual $^{5}$. Uno de ellos prometía la gestión de una reforma institucional significativa que incluiría la creación de un Ministerio de Medio Ambiente con dos Subsecretarías, una dedicada a los recursos naturales y la biodiversidad y la otra a la gestión ambiental. Además, una Superintendencia de Fiscalización Ambiental y la creación de un Servicio Nacional de áreas Protegidas. En el punto siete se comprometía a no incluir la opción nuclear en la política energética nacional. Otro punto era la promesa de una Ley Marco de Ordenamiento Territorial, indispensable para la gestión del litoral y de las cuencas hidrográficas. Con respecto al compromiso presidencial de dotar al país de una nueva institucionalidad en materia Medio Ambiental, durante su gobierno el Congreso Nacional aprobó el proyecto de Ley que crea el Ministerio de Medio Ambiente, el Servicio de Evaluación Ambiental, la Superintendencia de Fiscalización Ambiental y los Tribunales Ambientales.

A pesar de estas medidas legislativas, solamente una parte de los compromisos de gobierno se concretaron. Causarían alarma, preocupación y rechazo en la comunidad acciones contrarias a la trayectoria fijada, como la aprobación del Proyecto Pascua Lama (2006), la formación de un grupo de trabajo en Núcleo-Electricidad (2007), con la misión de asesorar al Gobierno en la evaluación de los estudios tendientes a la identificación de oportunidades, ventajas, desafíos y riesgos que involucraría el uso de la energía nuclear para la producción de electricidad en nuestro país ${ }^{6}$.

En beneficio de los Pueblos Indígenas, se promulgó la Ley que crea el Espacio Costero Marino de los Pueblos Originarios (Ley $\mathrm{N}^{\circ}$ 20.249) publicada en el Diario Oficial en el año 2008. El $1^{\circ}$ de abril del mismo año, se aprobó la política Re-Conocer: Pacto Social por la Multiculturalidad que priorizaría los derechos de estos pueblos, señalando que su cumplimiento no sólo es responsabilidad exclusiva del Gobierno sino también de la sociedad en su conjunto, haciéndolos transversales e institucionalizando el tema en toda la estructura estatal para la aplicación del Convenio 169 de la OIT, promulgado en el mes de septiembre de 2009.

En julio de 2008 fue promulgada la Ley Sobre Recuperación Del 
Bosque Nativo y Fomento Forestal (Ley N 20.283) luego de 15 años de espera. La Agrupación de Ingenieros Forestales por el Bosque Nativo (AIFBN) declaró como factores claves para el éxito de su aplicación la voluntad política y la entrega de recursos asociados. A dos años de la publicación en el Diario Oficial de la nueva ley, la AIFBN estimaba que las señales de las nuevas autoridades gubernamentales eran débiles, por lo que había que estar atentos para que efectivamente se manejasen los bosques nativos en concordancia con el desarrollo de las comunidades que los habitaban (Neira 2010). Efectivamente, tanto la Ley de Bosque Nativo, como el Reglamento de la Ley $N^{\circ} 20.283$ aprobado por el Congreso en septiembre de 2009, han sido criticados principalmente por el contenido ambiguo del artículo $\mathrm{N}^{0} 19$ y el explícito artículo $\mathrm{N}^{\mathrm{0}} 13$ del Reglamento, que facultan la corta o intervención de bosque nativo con fines de producción maderera o no maderera (PNM).

La Ley del Bosque Nativo, en su versión actual, no considera acciones que promuevan los servicios ecosistémicos tales como aumento de las oportunidades de recreación, conservación de suelo y agua, e incremento del abastecimiento de agua para consumo humano y otras actividades productivas, entre otras. Por otra parte, las bonificaciones a la plantación con especies nativas están concebidas entre las actividades bonificables para producción de madera y PNM o en los bosques de preservación, definidos como aquellos en que están presentes especies vegetales en las categorías de conservación (Lara, Urrutia, Little y Martínez, 2010: 5).

\section{Ley Nº 19.300 Sobre Bases Generales del Medio Ambiente}

La ley $N^{\circ} 19.300$ sobre Bases Generales del Medio Ambiente fue publicada en el Diario Oficial el 9 de marzo de 1994, habiendo ingresado en el Senado para su tramitación en septiembre de 1992. En el Título I de las Disposiciones Generales, artículo $1^{\circ}$, la ley prescribe el derecho a vivir en un medio ambiente libre de contaminación, la protección del medio ambiente, la preservación de la naturaleza y la conservación del patrimonio ambiental. En el artículo $2^{\circ}$, se aplica a las definiciones legales, de manera que obliga a los ciudadanos, autoridades y jueces a entender los términos en la forma que el legislador ha determinado. El modelo en que se basó la ley fue la Propuesta de Ley Básica de Protección Ambiental y Promoción del Desarrollo Sostenible elaborado para Latinoamérica por el Programa de las Naciones Unidas para el Medio Ambiente (PNUMA) de 1993.

En la ley se establecen diversos instrumentos de gestión ambiental, tales como el Sistema de Evaluación de Impacto Ambiental (SEIA) de los proyectos de inversión públicos y privados, la Declaración de Impacto Ambiental (DIA), y el Estudio de Impacto Ambiental (EIA), que es un documento más complejo, aplicable fundamentalmente a grandes proyectos de inversión, que pueden tener un impacto significativo en el medio ambiente y cuyos potenciales efectos no están regulados en su totalidad o dependen de apreciaciones de carácter subjetivo (por ejemplo la alteración 
de paisajes con valor patrimonial o los efectos sobre una comunidad y su cultura, etc.) Tanto la Declaración como el Estudio de Impacto Ambiental los realiza el titular del proyecto que será evaluado posteriormente por la Comisión Nacional del entidad que integran todos los servicios con competencias ambientales en cada región.

Del Fávero (1994: 2) acota muy bien que la ley no pretende cubrir todas las materias que dicen relación con el medio ambiente, pues se requerirá de leyes especiales para regular en detalle aquellas áreas que presentan particulares complejidades (piénsese en una ley de permisos de emisión transables, ley de suelos, ley forestal, etc.). Como señala su nombre, se trata de una ley de bases generales, que contempla lo más fundamental a fin de iniciar un proceso ordenador de la normativa ambiental en el país. Ella viene a establecer un mecanismo que posibilite la integración entre la regulación y política ambiental con la sectorial. El artículo 72 letra d) lo pone en claro al disponer que corresponderá al Consejo Directivo de la CONAMA proponer al Presidente de la República proyectos de ley y actos administrativos relativos a materias ambientales, sin perjuicio de las funciones propias de otros organismos públicos.

Guiloff (2011: 234) señala que la disposición mencionada (art. 72 letra f) fue interpretada en la práctica como una necesaria integración de los organismos sectoriales para la dictación de la regulación ambiental, dejando a la CONAMA sólo una labor de coordinación, lo que a su juicio, resultó pernicioso, y causal de los muchos avatares en que se ha visto complicado el control de la regulación ambiental en Chile, debido a las dificultades en la integración de las consideraciones o políticas sectoriales, lo que no pasó inadvertido en la evaluación que hiciera la OCDE el año 2005 y repercutió severamente sobre la gestión y la fiscalización. Al considerar que uno de los cimientos de la ley $\mathrm{N}^{\mathrm{o}} 19.300$ era, precisamente, la transversalidad en el ámbito de la regulación, de modo que toda norma o política ambiental que se apruebe implique equilibrar objetivos e intereses diversos, sin embargo, debido a la inexistencia de una normativa integradora en la ley, las autoridades sectoriales en vez de someter a deliberación una política o un hecho concreto, se limitaron a vetar. Para salvar este impasse se impone como absolutamente necesario, de acuerdo a este planteamiento, que la regulación de cada una de estas autoridades deba ser sometida al pronunciamiento del órgano transversal.

\section{Legislación Internacional sobre el Medio Ambiente y la Biodiversidad}

Conforme a la Constitución chilena, es el Presidente de la República quien concentra y monopoliza la negociación internacional ${ }^{7}$. El Jefe de Estado conduce las relaciones políticas con las potencias extranjeras y los organismos internacionales, y lleva a cabo negociaciones; concluye, firma y ratifica los tratados internacionales que estime convenientes para el país. 
Es rol del Congreso aprobar o desechar los tratados internacionales que le presentare el Presidente de la República antes de su ratificación, y la obligatoriedad del tratado en el plano interno sólo existe desde su publicación en el Diario Oficial (Llanos, 2003).

Son numerosos los Tratados Internacionales con incidencia ambiental suscritos por Chile y en vigencia, entre los cuales analizaremos -por su relación con el derecho consuetudinario de los pueblos indígenas a la utilización de los recursos naturales en sus territorios- el Convenio sobre zonas húmedas de Importancia Internacional, especialmente como hábitat de aves acuáticas (RAMSAR) (1981), y el Convenio sobre la Diversidad Biológica (1994).

En el documento Informe de la Evaluación de los Ecosistemas del Milenio, se definen tres problemas principales relacionados con nuestra gestión de los ecosistemas del mundo que son perjudiciales para la humanidad, y que disminuirán significativamente los beneficios que obtenemos de los ecosistemas a largo plazo. Aproximadamente un 60\% de los ecosistemas se están degradando o se usan de manera no sostenible, tales como el agua dulce, la pesca de captura, la purificación del aire y del agua, la regulación del clima regional y local, los riesgos naturales y las pestes, cuyos costes totales van en aumento (para alimentar a otros servicios), y la degradación suele desplazarse de un grupo de personas a otro, o traspasan los costos a las generaciones futuras. Se ha observado la probabilidad de que los cambios en los ecosistemas no sean lineales y se produzcan cambios acelerados, abruptos, y potencialmente irreversibles tales como las alteraciones bruscas de la calidad del agua, la creación de zonas muertas en las aguas costeras, el colapso de las pesquerías y los cambios en los climas regionales. Por último el Informe señala que la degradación de los servicios de los ecosistemas está contribuyendo al aumento de las desigualdades entre los grupos de personas o comunidades y es el principal factor causante de la pobreza y del conflicto social.

La inconsistencia entre las normas internacionales que imponen los Tratados suscritos por Chile y en vigencia, con la débil normativa interna en legislación ambiental -y el derecho constitucional de las personas a un medio ambiente libre de contaminación- queda de manifiesto en un caso emblemático como ha sido el del Santuario de la Naturaleza Río Cruces impactado por las descargas altamente contaminantes de la empresa Celulosa Arauco y Constitución (CELCO) ${ }^{8}$ y la masiva extinción y migración de cisnes de cuello negro y taguas.

Una posición que debiera guiar la política medioambiental chilena -y que comparten los académicos investigadores (Lara et al., 2010)- es la de Dourojeanni (1999:24), quien recomienda conocer a fondo el sistema de agua superficial y subterránea antes de seguir explotándolos; y subraya que una de las acciones primordiales que se deben llevar a cabo es crear instancias de gestión del agua por cuenca, o sistema hídrico interconectado 
adecuados a cada lugar, de manera que la Dirección General de Aguas (DGA) repose en estas organizaciones para poder conocer las situaciones, investigar y entregar una información adecuada a los usuarios del agua. Además es la única forma de comprometer a los propios usuarios en la gestión de un recurso compartido.

\section{Los Humedales chilenos y el Convenio sobre zonas húmedas de Importancia Internacional, especialmente como hábitat de aves acuáticas (RAMSAR)}

La Convención de RAMSAR sobre zonas húmedas como hábitat de aves acuáticas fue firmada por Chile el 2 de febrero de 1971, en Irán, y promulgada en noviembre de 1981 por el Ministerio de Relaciones Exteriores. Este convenio persigue detener la desaparición de los humedales en el mundo y la pérdida de funciones que cumplen los distintos ecosistemas relacionados por los flujos de agua. El documento enfatiza el rol de los humedales como un recurso de gran valor económico, cultural, científico y recreativo, cuya pérdida sería irreparable. Ellos tienen funciones ecológicas fundamentales como reguladores de los regímenes hidrológicos y como hábitat de una flora y fauna características, especialmente de aves acuáticas que deben ser consideradas un recurso internacional, puesto que en sus migraciones estacionales atraviesan las fronteras.

En Chile existen doce sitios naturales formando parte de la red internacional de humedales de importancia para la conservación de la diversidad biológica mundial y el sustento de la vida humana, a los que se agregan últimamente los salares de Puisa de San Pedro de Atacama y el de Aguas Calientes IV de Antofagasta. Del total de humedales protegidos (lista Ramsar) solamente dos se encuentran en la zona austral: el de Bahía Lomas en la región de Magallanes, y el Santuario Carlos Anwandter ${ }^{9}$ en la Región de Los Ríos.

La insuficiencia en el número de humedales protegidos en Chile queda de manifiesto ante la cantidad de sitios de interés de conservación dados a conocer en el documento Estrategia Regional para la Conservación y Utilización Sostenible de la Biodiversidad; Décima Región de Los Lagos (CONAMA, 2002), en el que figuran 66 sitios de interés de conservación, solamente en esta región. Entre los sitios clasificados en el Nivel 1, de alto grado de interés y factibilidad de protección, se encuentra Curiñanco, que es un área orientada a conservar los escasos bosques de olivillo costero (Aetoxicum punctatum) y mamíferos marinos, presentes al Norte de la ciudad de Valdivia. También está el Parque Nacional Chiloé, al cual se propone agregar sistemas de alto interés como el humedal del Lago Chepu y el Lago Huillinco, este último con importantes poblaciones de aves marinas que lo utilizan como área de paso y refugio durante distintos periodos del año. 


\section{La protección de la biodiversidad en Chile y el Convenio sobre la Diversidad Biológica}

Durante la Cumbre de la Tierra en Río de Janeiro el año 1992, fue firmado el Convenio sobre la Diversidad Biológica ${ }^{10}$ de la Organización de las Naciones Unidas por 150 mandatarios y líderes gubernamentales asistentes. Chile lo ha incorporado a la legislación interna mediante el D.S. N ${ }^{\circ}$ 1.963 de 1994, y publicado en el Diario Oficial el 6 de junio de 1995.

En este documento, las Naciones Unidas reconoce -como la exigencia fundamental para detener la pérdida de diversidad- la conservación in situ de los ecosistemas y hábitats naturales y el mantenimiento y la recuperación de poblaciones viables de especies en sus entornos naturales, así como la estrecha y tradicional dependencia de muchas comunidades locales y poblaciones indígenas que tienen sistemas de vida tradicionales basados en los recursos biológicos, y la conveniencia de compartir equitativamente los beneficios que se derivan de la utilización de los conocimientos tradicionales, las innovaciones v las prácticas pertinentes para la conservación de la diversidad biológica y la utilización sostenible de sus componentes. Subraya también el rol que desempeña la mujer en la conservación y la utilización sostenible de la diversidad biológica, y la importancia de la cooperación internacional, regional y mundial entre los Estados y las organizaciones intergubernamentales y el sector no gubernamental para la conservación de la diversidad biológica y la utilización sostenible de sus componentes.

En Chile, el Sistema Nacional de Áreas Silvestres Protegidas del Estado (SNASPE), es el principal instrumento en materia de conservación de la biodiversidad, junto a otros cuerpos normativos dispersos y manejados en diferentes ministerios y servicios públicos. El SNASPE es un programa ejecutado y administrado por la Corporación Nacional Forestal (CONAF) que cubre el 19\% del territorio aproximadamente, habiéndose definido 127 ecosistemas terrestres (según sus características vegetacionales) de los cuales 86 presentan menos del 10\% de su superficie bajo protección, 47 menos del $1 \%$ y 25 se encuentran sin cobertura ${ }^{11}$. A pesar de estas cifras que demuestran la desprotección de los ecosistemas, la Comisión Nacional de Medio Ambiente (CONAMA) hace entrega de un cuadro poco realista en su Cuarto Informe sobre Biodiversidad de 2009. Hay consenso en que un elemento decisivo a la hora de implementar las políticas de protección de los recursos naturales es el presupuesto que se le asigna. Todos los estudios y análisis -incluso gubernamentales- sostienen que existe incoherencia entre la agenda ambiental del país que se proyecta y el financiamiento (Barton, Reyes y Galilea, sin año).

En diciembre de 2003 se aprueba la Estrategia Nacional de Biodiversidad (ENBD) la cual es la primera política pública que integra la visión de los distintos organismos estatales y las preocupaciones de las organizaciones sociales y gremiales, en relación con la biodiversidad en todos sus niveles. En el informe sobre la Estrategia Nacional de 
Biodiversidad (CONAMA, 2003) y en la Estrategia de Biodiversidad de la Región de Los Lagos (2002), se encarece el respeto por los conocimientos tradicionales, nombrando como actores relevantes en la X Región, entre otros, a CONADI y las Comunidades Indígenas en los planes de acción. La participación de las comunidades indígenas en las Áreas Silvestres Protegidas del Estado (ASPE) no ha sido considerada en la práctica, a pesar de que el artículo 35 de la Ley Indígena promulgada en 1993 establece que los organismo del Estado deben considerar la participación de los indígenas al interior de las áreas silvestres protegidas que se encuentren en las ADI, así como regular los derechos de uso que corresponden a las comunidades (Aylwin, 2002).

\section{Discusión y Conclusiones}

Peña, Luraschi y Valenzuela (2004: 48) afirman que en el caso de Chile, no cabe duda que la institucionalidad vigente es el resultado de la evolución compleja de las fuerzas presentes en la sociedad y de los desafíos que van surgiendo junto al crecimiento, y no el resultado de un diseño único y una estructura concebida coherentemente desde el primer momento. Esto explicaría las dificultades que ha enfrentado la Ley de Bases Generales del Medio Ambiente para garantizarnos el derecho a vivir en un ambiente libre de contaminación, asegurar la protección y conservación de los recursos naturales, la biodiversidad y los derechos indígenas.

Van Bebber (2002: 327) sostiene que las presiones internas al modelo de Estado surgen especialmente de una puesta en cuestión de la idea de nación homogénea que sustenta al proyecto cultural del EstadoNación chileno. Es en esta perspectiva donde se sitúa la dimensión política del fenómeno de la etnicidad, como un desafío radical a la construcción histórica de la idea de Nación en Chile. En Stuven (2000, 29) se develan los valores, las ideas de la clase dirigente, la elite que dio forma y contenido al Estado-Nación. La investigadora propone que "para la clase chilena en torno a 1810, institucionalizar un Estado republicano, con separación de poderes, régimen representativo, y reconocimiento del concepto de soberanía popular como inherente a él, no implicaba necesariamente la aceptación y menos la puesta en práctica de las consecuencias de democratización social e inclusión política que esos conceptos traían consigo”.

Transcurridas casi dos décadas de gobiernos democráticos en Chile, el 19\% de la población indígena vivía al 2006 bajo la línea de pobreza en contraste con el 13,7\% de la población no indígena. La Ley N $\mathrm{N}^{\mathrm{O}} 19.253$ de 1993 sobre Fomento, Protección y Desarrollo de los indígenas, aún no recoge los lineamientos del derecho internacional que les es aplicable, a ello se suma la Ley N N $^{0} 20.294$ que crea el Espacio Marino Costero de los Pueblos Originarios, promulgada el año 2008, y el Convenio $\mathrm{N}^{\mathrm{o}} 169$ de la OIT que entró en vigencia en septiembre de 2009. 
La Ley Sobre Recuperación del Bosque Nativo y Fomento Forestal, en vez de robustecer la eficacia reguladora de la CONAF, ha privatizado sus funciones y excluido del comité consultor a los pueblos indígenas, ambientalistas, trabajadores forestales y científicos, pudiendo oficializar la sustitución del bosque nativo por plantaciones de pino y eucaliptos. Con el acuerdo de ENDESA y del Banco Mundial, nuevos proyectos hidroeléctricos están en marcha, como el del río Futaleufu, río Puelo o Hydroaysén. En opinión de Altieri y Rojas (1999: 71) claramente, si se consideran estas tendencias, la economía chilena se mueve a lo largo de un trayecto insostenible, puesto que el crecimiento económico no toma en cuenta la disminución del capital natural y social del que la economía del futuro depende; el modelo propuesto olvida que los ecosistemas sostienen la economía, la economía no sostiene los ecosistemas. Así, el desafío para Chile en la nueva década (2000-2010) será encontrar caminos para sostener el crecimiento económico, promover la integración regional, y proteger el medioambiente a través de un modelo más equitativo de desarrollo.

Desde la perspectiva del análisis -en nuestro concepto- históricamente se conforma una política legislativa retórica, obsecuente con las empresas nacionales y extranjeras que invierten en la explotación de los recursos naturales, que no se condice con la realidad social, política, cultural, religiosa, y las necesidades de los pueblos que coexisten en el territorio, con la democracia e inclusión política de las mayorías, dictada desde una clase privilegiada - la elite criolla - que impone sus intereses y modelos de desarrollo de manera hegemónica. Grez (2009, 16) argumenta respecto a la ausencia de un poder democrático en Chile, señalando hitos de nuestra historia legislativa que así lo demuestran, a partir de los primeros ensayos constitucionales realizados durante la lucha por la independencia, haciendo presente que la emancipación política de Chile fue un acto eminentemente aristocrático de la clase dirigente que construyó el Estado nacional de acuerdo a sus intereses y concepción del mundo, asegurando su plena hegemonía y excluyendo de la vida política legal a la inmensa mayoría de la población. De la misma manera la Constitución del 80 fue elaborada y aprobada en los años más duros de la dictadura militar.

Un aporte significativo a la conceptualización del desarrollo sustentable, es el que hace Hervé (2010: 12) cuando analiza nuestra normativa ambiental tanto en la ley $\mathrm{N}^{\mathrm{o}} 19.300$, como en la ley $\mathrm{N}^{\circ} 20.417$ que viene a reemplazarla, haciendo notar que no se incorpora en ellas expresamente el concepto de justicia ambiental, complementario del desarrollo sustentable, y que en el derecho comparado e internacional constituyen la base sustantiva de las políticas públicas y la legislación ambiental. Recomienda que nuestro sistema jurídico debiera explicitar el deber del Estado de iniciar políticas activas para eliminar las diversas fuentes de discriminación ambiental. Y mientras esto no ocurra nuestra legislación estará dirigida principalmente a regular y eventualmente perseguir la responsabilidad de quienes contaminan, pero no a establecer un deber del Estado de suprimir estas 
situaciones de contaminación y degradación ambiental. Su opinión es que mientras se mantenga exclusivamente la referencia a la sustentabilidad sin enfatizar el elemento de la justicia, existirá una posición más bien pasiva y no se desarrollarán las herramientas necesarias para hacerse cargo de estos temas. 


\section{Notas}

${ }^{1}$ Este artículo es parte de los resultados del Proyecto FONDECYT № 1110570 “Cosmovisión huilliche y naturaleza: poética, discurso y políticas territoriales”.

${ }^{2}$ Report of the United Nations Conference on Environment and Development (Rio de Janeiro, 3-14 June 1992).

${ }^{3}$ En 1970 Rafael Elizalde publica La Sobrevivencia de Chile. La Conservación de sus Recursos Naturales Renovables (Segunda Edici6n). Ministerio de Agricultura. Servicio Agrícola y Ganadero. Ed. El Escudo. Santiago de Chile. Donde hace notar que junto con la destrucción de los recursos naturales renovables, la degradación del medio ambiente es uno de los factores de mayor peligrosidad para la supervivencia de esos mismos recursos.

${ }^{4}$ Durante 44 años, Endesa Chile perteneció al Estado de Chile. El proceso de privatización comenzó en 1987, a través de una serie de ofertas públicas de acciones; y fue completado en 1989.

${ }^{5}$ El "Acuerdo de Chagual" fue firmado a fines del 2005 por 23 organizaciones no gubernamentales con Bachelet.

${ }^{6}$ Balance Programático Michelle Bachelet 2006-2010. Resumen. Capturado el 3 de junio 2012 en http://www.sernac.cl/acercade/Resumen\%20Program\%C3\%A1tico\%2020062010.pdf.

${ }^{7}$ Constitución Política de la República de Chile (2005).

${ }^{8}$ Celulosa Arauco y Constitución (CELCO) es una sociedad anónima constituida en septiembre de 1979 como resultado de la fusión de Celulosa Arauco S.A. (1967) y Celulosa Constitución S.A. (1969), ambas empresas de la CORFO privatizadas respectivamente en 1977 y 1979.

${ }^{9}$ El Santuario de la Naturaleza fue creado legalmente en 1981 mediante D.S. N ${ }^{0} 2734$ del Ministerio de Educación. En 1984 fue escogido por Chile para subscribir la Convención Relativa a los humedales de Importancia Internacional, especialmente como hábitat de aves acuáticas.

${ }^{10}$ Convenio Sobre La Diversidad Biológica, Río de Janeiro, Brasil, 5 de junio de 1992. Capturado el 5 de abril 2012 en http://www.iadb.org/Research/legislacionindigena/pdocs/ CONVENIODIVERSIDAD.pdf.

${ }^{11}$ SNASPE (2005). Síntesis Ejecutiva No 13. Programa Sistema Nacional de Áreas Silvestres Protegidas SNASPE. 


\section{Bibliografía}

Altieri, Miguel y Alejandro Rojas (1999), “Ecological Impacts Of Chile’s Neoliberal Policies, With Special Emphasis On Agroecosystems”, en Environment, Development and Sustainability N¹: 55-72, Kluwer Academic Publishers. Netherlands.

Aylwin, José (2000), “Antecedentes para la comprensión de los conflictos en el territorio mapuche”, Revista Perspectivas, N³ (2), Universidad de Chile.

Idem (2002 ), "Políticas Públicas y Pueblos Indígenas: El Caso De la Política de Tierras del Estado Chileno y el Pueblo Mapuche”. En Desarrollo Comunitario Auto sostenible en una Perspectiva Comparada (CLASPO), (capturado el 8 de mayo 2012 en http://lanic.utexas.edu/project/laoap/ claspo/dt/0006.pdf).

Catalán, Rodrigo y Ramos, Ruperto (1998), “Los Bosques Nativos Del Sur De Chile Y El Pueblo Mapuche”, World Rainforest Movement (WRM). Boletín 13, (capturado el 2 de junio 2012 en http://www.wrm.org.uy/ plantations/information/Catalan.html).

Barton, Jonathan, Francisca Reyes, y Sergio Galilea (sin año), El Nuevo Diseño de la Institucionalidad Ambiental en Chile, (capturado el 28 de mayo 2012 en http://politicaspublicas.uc.cl/media/proyectos/material/ 474_El_Nuevo_Diseno_de_la_Institucionalidad_Ambiental_en_Chile.pdf

Bengoa, José (1996), Historia del Pueblo Mapuche (Siglos IX y X). Ed. Sur. Colección Estudios Históricos. 426 p.

Camus, Pablo, y Ernst R.Hajek (1998), Historia Ambiental De Chile, Andros Impresores, Santiago de Chile. 183 p.

Carrasco, Sergio (2008), “La evolución político-constitucional de Chile”, Estudios Constitucionales, Año 6, № 2, 2008: 301-324. Centro de Estudios Constitucionales de Chile, Universidad de Talca.

Castro, Milka (2003), “Desafíos de las Políticas Interculturales en Chile”. FERMENTUM 13 N³8:520-538 (septiembre - diciembre ) Mérida, Venezuela.

Catalán, Rodrigo y Ruperto Ramos (1998), “Los Bosques Nativos Del Sur De Chile Y El Pueblo Mapuche” World Rainforest Movement (WRM). Boletín $N^{\circ} 13$, (capturado el 2 de junio 2012 en http://www.wrm.org.uy/ plantations/information/Catalan.html).

CEPAL (1999), Debate sobre el Código de Aguas de Chile (capturado el 25 de marzo de 2012 en http://www.eclac.cl/publicaciones/xml/9/4489/ LCR1924-E.pdf 
CONAMA (2002 ), Estrategia Regional para la Conservación y Utilización Sostenible de la Biodiversidad; Décima Región de Los Lagos, (capturado el 15 de marzo de 2012 en http://www.mma.gob.cl/biodiversidad/1313/ articles-48849_EstrategiaRegionalBiodiversidadPDA_10.pdf).

Idem (2003), Estrategia Nacional de Biodiversidad, (capturado el 29 de marzo 2012 en http://www.mma.gob.cl/biodiversidad/1313/w3-article48802.html).

Idem (2009), Convenio Sobre Diversidad Biológica. Cuarto Informe Nacional De Biodiversidad Chile. (capturado el 5 de abril 2012 en http:// www.chiledesarrollosustentable.cl/desarrollo-sustentable/ministerio-demedio-ambiente/gestion/estrategia-nacional-de-biodiversidad/.)

CONAMA - ORPALC- CEPAL (2009), Informe País sobre Estado del Medio Ambiente en Chile . Centro De Análisis De Políticas Públicas. Instituto De Asuntos Públicos Universidad De Chile. Geo Chile.

Correa, Martín, Rodrigo Catalán y Martín Paillamanque (2001), Percepción de las comunidades huilliches sobre el proyecto ruta costera Sur. Editado por Francisco Morey Cañoles, (capturado el 8 de abril 2012 en http://ccc2.cl.tripod.com/edit42.PDF)

Del Fávero, Gabriel (1994), “Ley Sobre Bases Generales Del Medio Ambiente”. Estudios Públicos N54: 1-46 (otoño)

Dourojeanni, Axel (1999), “Debate sobre el Código de Aguas de Chile”. Comisión Económica para América Latina (CEPAL) (capturado el 9 de abril 2012 en http://www.eclac.cl/publicaciones/xml/9/4489/LCR1924E.pdf)

Figueroa, Verónica (2009),“Desarrollo indígena y políticas públicas: Una difícil relación para una convivencia multicultural”, Docencia No 37 . (capturado el $1^{\circ}$ de junio 2012 en http://www.revistadocencia.cl/pdf/ 20100730182845.pdf)

Foerster, Rolf, y Jorge Vergara (2000), "Los mapuches y la lucha por el reconocimiento en la sociedad chilena”. En XII Congreso Internacional. Derecho consuetudinario y pluralismo legal: desafíos en el tercer milenio N¹ :191-206, editado por Milka Castro

Frêne, Cristian, y Mariela Núñez (2010) "Hacia un nuevo Modelo Forestal en Chile”. Bosque Nativo $\mathrm{N}^{\circ}$ 47: 25 - 35

Grez, Sergio (2009), La Ausencia de un Poder Constituyente Democrático en la Historia de Chile”. Tiempo histórico N¹, Santiago de Chile, Universidad Academia de Humanismo Cristiano, (junio).

Guiloff, Matías (2011), “Nueva Institucionalidad Ambiental: Hacia Una 
Regulación Deliberativa”. Anuario De Derecho Público Universidad Diego Portales. Santiago de Chile.

Hajek, Ernst, Patricio Gross. y Guillermo Espinoza (1990), Problemas Ambientales De Chile. Agencia Internacional para el Desarrollo (AID) (1): $1-232$

Hervé, Dominique (2010), “El Desarrollo Sustentable y la Justicia Ambiental en la Ley 19.300 y en el Proyecto de Reforma”. Reforma a la Institucionalidad Ambiental. Antecedentes y Fundamentos: 265-288. Facultad de Derecho, editor Universidad Diego Portales. Santiago de Chile

Lara, Antonio, Rocío Urrutia, Christian Little y Andrés Martínez (2010), “Servicios Ecosistémicos y Ley del Bosque Nativo: No basta con definirlos”. Revista Bosque Nativo N 47: 3 - 9 .

Llanos, Hugo (2003), “Los Tratados Internacionales en la Constitución de 1925 y en la Jurisprudencia”. Artículos de Doctrina. Ius et Praxis N9:1 Talca 2003

Morales, Roberto, Marco Tamayo y Martín Cox (2010), “Pueblos Indígenas, Recursos Naturales Y Compañías Multinacionales: Hacia Una Convivencia Responsable Estudio de casos: Pueblo Mapuche Williche de Chiloé (Chile), Pueblo Shuar de la provincia de Zamora (Ecuador) y Comunidades Indígenas (Canadá)”. Informe De Investigación. Serie Avances de Investigación $\mathrm{N}^{\circ}$ 40. Escuela de Antropología, Universidad Austral de Chile. Fundación Carolina, España

Neira, Eduardo (2010), “Solidez, Coherencia y Consecuencia” (Editorial) Revista Bosque Nativo N 46 (febrero-junio)

Olivares Alberto (2010), "El Nuevo Marco Institucional Ambiental En Chile”, Revista Catalana De Dret Ambiental, N¹: 1 -23

Peña, Humberto, Marco Luraschi y Soledad Valenzuela (2004), “Agua, desarrollo y políticas públicas: la experiencia de Chile”. REGA 1 N²: 2550 (julio)

RAMSAR (1971), Convención Relativa a los Humedales de Importancia Internacional. Especialmente como Hábitat de Aves Acuáticas (1994), Ramsar, Iran, 2.2.1971.

Reid Walter, Harold A. Mooney, Angela Cropper, Doris Capistrano (2005), Evaluación de los Ecosistemas del Milenio Informe de Síntesis, editado por PNUMA, Caracas, Venezuela.

Reyes Bernardo, y Daniela Jara (sin año), Gobernanza Ambiental: Mensajes Desde La Periferia Instituto de Ecología Política. IEP :1-20. Santiago de Chile (capturado el 3 de junio 2002 en http://www.grupochorlavi.org/ 
gobernanzaambiental/Completos/IEP.pdf)

Rojas, Alejandro, Francisco Sabatini y Claudia Sepúlveda (2003), “Conflictos ambientales en Chile: aprendizajes y desafíos”. Revista Ambiente $Y$ Desarrollo de CIPMA, N¹9: 22-30

Smith, Sara (2005), La ‘cuestión indígena’ y el Estado Nacional: análisis crítico de discursos oficiales contemporáneos en Chile. Tesis de Maestría de Sara Université de Montreal,

Stuven, Ana Maria (2000), La seducción de un orden. Las élites y la construcción de Chile en las polémicas culturales y políticas del siglo XIX, Ediciones Universidad Católica de Chile, Santiago de Chile.

Toledo, Víctor (2003), Ecología, Espiritualidad y Conocimiento -de la sociedad del riesgo a la sociedad sustentable-, Programa de las Naciones Unidas para el Medio Ambiente, Oficina Regional para América Latina y el Caribe, México D. F.

Uriarte, Edume (2006), Estado, Gobierno, Nación. Tres conceptos fundamentales de la política. Pontificia Universidad Católica de Chile. Facultad de Historia, Geografía y Ciencia Política. Curso- Taller (4): 1-13)

Van Bebber, Rodrigo (2002), Estado-Nación y "conflicto mapuche": aproximación al discurso de los partidos políticos chilenos. Ed. Taller de Gráficas y Servicios S.R.L. Argentina.

Recibido: 14.10.2012

Aceptado: 16.01.2013 